\title{
HIGHLIGHT
}

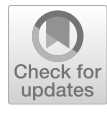

Cite as

Nano-Micro Lett.

(2021) 13:210

Received: 7 September 2021

Accepted: 12 September 2021

Published online: 16 October 2021

(C) The Author(s) 2021

\section{Separator Wettability Enhanced by Electrolyte Additive to Boost the Electrochemical Performance of Lithium Metal Batteries}

\author{
Ying Wang ${ }^{1 凶}$
}

Lithium (Li) metal has been regarded as one of the most promising candidates to replace graphite anode due to its high theoretical specific capacity and the lowest electrochemical potential [1-3]. However, the immoderate growth of $\mathrm{Li}$ dendrite during Li plating/stripping causes serious safety problem and poor performance that severely impedes the practical application of lithium metal batteries (LMBs) [4-6]. Until now, there have been numerous kinds of strategies be proposed to inhibit Li dendrites growth and protect lithium metal anode such as high concentration electrolytes [7], construction of the solid electrolyte interface layer [8], structural design of anode materials [9], regulation of $\mathrm{Li}^{+}$solvation [10], and solid-state electrolytes [11]. As an important part of battery structure, separator plays a vital role in the performance of battery [12]. The main function of separator is to divide the anode and cathode that prevents internal short circuit caused by direct contact between anode and cathode. So, the separator needs to be electrically insulated. At the same time, the separator also needs to ensure that the electrolyte is conductive between anode and cathode [13]. Therefore, it is necessary to render the separator fully wetted. Nevertheless, there are few researches on enhancing the wettability of the separator especially functional electrolyte additives.

Recently, Ma's group conducted a detailed research and discussion on the study of separator wettability [14]. They employed heptafluorobutyric anhydride (HFA) as a multifunctional additive to modify the commercial electrolyte (1 M $\mathrm{LiPF}_{6}$ in EC/DMC 1:1). Benefited by the special chain structure, HFA can serve as the surfactant to promote the wetting of separator. Good wettability can make the separator easily to be wetted that facilitates the permeation of electrolyte. As shown in Fig. 1a, the schematic diagram visually describes the effect of different separator wettabilities toward electrolyte on $\mathrm{Li}^{+}$transportation. The electrolyte must entirely fill the holes in the separator so that the channels for $\mathrm{Li}^{+}$transferring can be built. The poor wettability of the electrolyte will cause some invalid channels in the separator that result in uneven $\mathrm{Li}^{+}$flux for the whole Li metal surface. To assess the wettability, Ma's group carried out the electrolyte uptake test and calculated the degree of electrolyte filling. After adding $1.0 \mathrm{wt} \%$ HFA, the electrolyte can wet the separator immediately, while the blank electrolyte forms into a droplet after dropping on the surface of the separator, as shown in Fig. 1b. In addition, the HFA-contained electrolyte uptake is $92.1 \%$, much higher than $10.5 \%$ for blank electrolyte uptake. The degree of electrolyte filling increases from $11.1 \%$ in blank electrolyte to $97.3 \%$ in HFA-contained electrolyte, implying that the holes in separator have been sufficiently filled to build continuous pathways for $\mathrm{Li}^{+}$flux. The poor wettability of separator also causes a higher resistance, resulting from the blocked paths for $\mathrm{Li}^{+}$ transportation (Fig. 1c). More intuitively, the introduction of HFA can reduce the surface tension of the electrolyte, reflecting in the smaller contact angle of electrolyte dropping on the separator, from $65.4^{\circ}$ in blank electrolyte (Fig. 1d) reduce to $40.5^{\circ}$ in HFA-contained electrolyte (Fig. 1e).

The sufficient $\mathrm{Li}^{+}$transport channels can render uniform Li-ion flux, making the deposition of the lithium more

Ying Wang, ying.b.wang@cuhk.edu.hk

1 Department of Chemistry, The Chinese University of Hong Kong, Shatin, N. T., Hong Kong SAR, People's Republic of China 

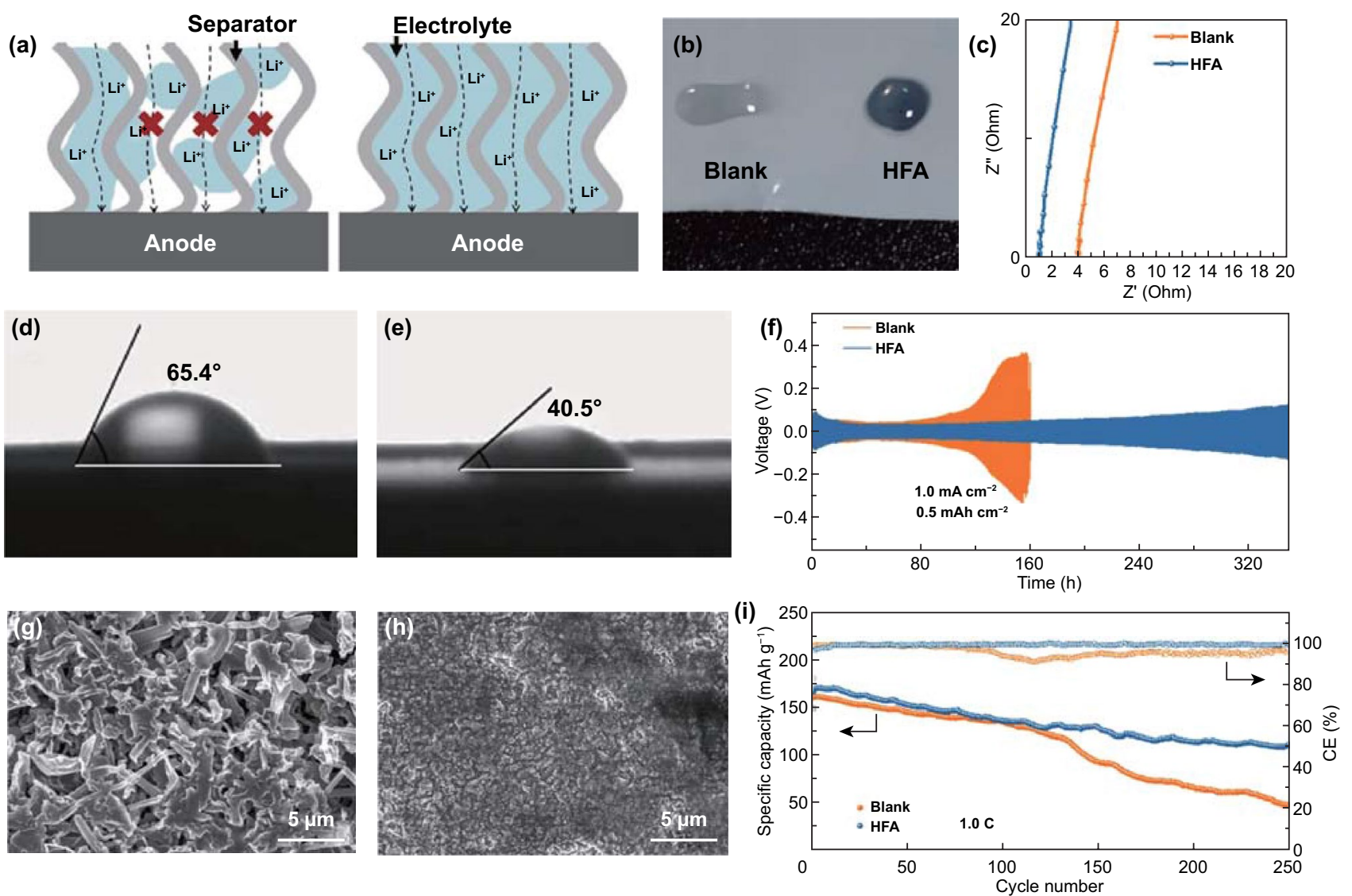

Fig. 1 a Schematic illustration of the impacts of separator wettability toward electrolyte on $\mathrm{Li}^{+}$transportation. $\mathbf{b}$ The photograph of different electrolytes dropped on the separator. c EIS result of SSIISS symmetric cells in different electrolytes. $\mathbf{d}$ The contact angles on separator for blank electrolyte. e The contact angles on separator for $1.0 \mathrm{wt} \%$ HFA-contained electrolyte. $\mathbf{f}$ The cycle performance of LillLi symmetric cells in different electrolytes. g SEM image of Li anode after 50 cycles in blank electrolyte. h SEM images of Li anode after 50 cycles in 1.0 wt\% HFAcontained electrolyte. i Cycling performance of LillNCM622 cells in different electrolytes

uniform in the surface of anode. This will inhibit the growth of Li dendrites. As shown in Fig. 1f, the LillLi symmetric cells assembled with HFA-contained electrolyte have excellent life performance that steadily cycles more than $320 \mathrm{~h}$ without severe polarization, while the cells with blank electrolyte rapidly fail only after $120 \mathrm{~h}$. In addition, the surface of $\mathrm{Li}$ anode after 50 cycles in blank electrolyte is full of needle-like Li dendrites (Fig. 1g). In sharp contrast, there is no Li dendrite on the surface of anode cycling in HFA-contained electrolyte (Fig. 1h), implying a uniform deposition of lithium. Moreover, the addition of HFA also improves the cyclic stability performance of LillNCM622 full cells, rendering higher capacity retention and Coulombic efficiency (CE), as shown in Fig. 1i.

In summary, this work from Ma's group systematically and comprehensively explained the influence of separator wettability toward battery performance. The study on ion flux can also become a new research direction for LMBs to inhibit the growth of dendrite. In addition, they proposed the concept of electrolyte filling degree in separator, which could be a new index to study electrolytes in future.

Open Access This article is licensed under a Creative Commons Attribution 4.0 International License, which permits use, sharing, adaptation, distribution and reproduction in any medium or format, as long as you give appropriate credit to the original author(s) and the source, provide a link to the Creative Commons licence, and indicate if changes were made. The images or other third party material in this article are included in the article's Creative Commons licence, unless indicated otherwise in a credit line to the material. If material is not included in the article's Creative Commons licence and your intended use is not permitted by statutory regulation or exceeds the permitted use, you will need to obtain permission directly from the copyright holder. To view a copy of this licence, visit http://creativecommons.org/ licenses/by/4.0/. 


\section{References}

1. S. Xia, X. Wu, Z. Zhang, Y. Cui, W. Liu, Practical challenges and future perspectives of all-solid-state lithium-metal batteries. Chem 5(4), 753-785 (2019). https://doi.org/10.1016/j. chempr.2018.11.013

2. X. Shen, H. Liu, X. Cheng, C. Yan, J. Huang, Beyond lithium ion batteries: higher energy density battery systems based on lithium metal anodes. Energy Stor. Mater. 12, 161-175 (2018). https://doi.org/10.1016/j.ensm.2017.12.002

3. X. Cheng, R. Zhang, C. Zhao, Q. Zhang, Toward safe lithium metal anode in rechargeable batteries: a review. Chem. Rev. 117(15), 10403-10473 (2017). https://doi.org/10.1021/acs. chemrev.7b00115

4. N. Wu, Y. Li, A. Dolocan, W. Li, H. Xu et al., In situ formation of $\mathrm{Li}_{3} \mathrm{P}$ layer enables fast $\mathrm{Li}^{+}$conduction across $\mathrm{Li} /$ solid polymer electrolyte interface. Adv. Funct. Mater. 30(22), 2000831 (2020). https://doi.org/10.1002/adfm.202000831

5. D. Lin, Y. Liu, Y. Cui, Reviving the lithium metal anode for high-energy batteries. Nat. Nanotechnol. 12, 194-206 (2017). https://doi.org/10.1038/nnano.2017.16

6. S. Bai, X. Liu, K. Zhu, S. Wu, H. Zhou, Metal-organic framework-based separator for lithium-sulfur batteries. Nat. Energy 1, 16094 (2016). https://doi.org/10.1038/nenergy.2016.94

7. X. Fan, L. Chen, X. Ji, T. Deng, S. Hou et al., Highly fluorinated interphases enable high-voltage Li-metal batteries. Chem 4(1), 174-185 (2018). https://doi.org/10.1016/j.chempr. 2017.10.017

8. F. Li, J. He, J. Liu, M. Wu, Y. Hou et al., Gradient solid electrolyte interphase and lithium-ion solvation regulated by bisfluoroacetamide for stable lithium metal batteries. Angew. Chem. Int. Ed. 60(12), 6600-6608 (2021). https://doi.org/10. 1002/anie.202013993

9. Y. Feng, C. Zhang, B. Li, B. Li, S. Xiong et al., Low-volumechange, dendrite-free lithium metal anodes enabled by lithophilic 3D matrix with LiF-enriched surface. J. Mater. Chem. A 7(11), 6090-6098 (2019). https://doi.org/10.1039/C8TA1 0779C

10. H. Wang, J. He, J. Liu, S. Qi, M. Wu et al., Electrolytes enriched by crown ethers for lithium metal batteries. Adv. Funct. Mater. 31(2), 2002578 (2020). https://doi.org/10.1002/ adfm.202002578

11. T. Famprikis, P. Canepa, J.A. Dawson, M.S. Islam, C. Masquelier, Fundamentals of inorganic solid-state electrolytes for batteries. Nat. Mater. 18, 1278-1291 (2019). https://doi. org/10.1038/s41563-019-0431-3

12. M. Ryou, D.J. Lee, J. Lee, Y.M. Lee, J.K. Park et al., Excellent cycle life of lithium-metal anodes in lithium-ion batteries with mussel-inspired polydopamine-coated separators. Adv. Energy Mater. 2(6), 645-650 (2012). https://doi.org/10.1002/aenm. 201100687

13. H. Zheng, Y. Xie, H. Xiang, P. Shi, X. Liang et al., A bifunctional electrolyte additive for separator wetting and dendrite suppression in lithium metal batteries. Electrochim. Acta 270, 62-69 (2018). https://doi.org/10.1016/j.electacta.2018.03.089

14. J. Huang, J. Liu, J. He, M. Wu, S. Qi et al., Optimizing electrode/electrolyte interphases and Li-ion flux/solvation for lithium-metal batteries with qua-functional heptafluorobutyric anhydride. Angew. Chem. Int. Ed. 60(38), 20717-20722 (2021). https://doi.org/10.1002/anie.202107957 